\title{
Tips on preparing your medical CV
}

\author{
Kathy Oxtoby \\ London, UK
}

As a doctor, your CV represents the sum total of relevant medical activity in your career to date. It's not enough to list what you've done, though-you need to create a document that is clearly presented, fits the job specification, and highlights your achievements.

\section{Essential elements for a perfect CV}

There is no standard way to create a CV but there are common headings to include, such as personal details, education, and qualifications. Daniel Redfern, a consultant in trauma and orthopaedics at Lancashire Teaching Hospitals NHS Trust, recommends creating a content list which signposts the reader to sections that highlight your skills and experience, including career history, current position, and job title.

Your career history traditionally starts with your current position and then includes other posts you have held to date. Other sections that should be included are teaching, research, and managerial experience in whichever order is most relevant to the application.

Redfern also suggests adding a section on quality improvement, including any audit work, as well as one on presentations and publications. This can be divided into papers, invited chapters, and podium presentations, and can be grouped into international, national, and regional work for ease of reading.

More controversial is whether to include a personal statement. Redfern says that views vary from specialty to specialty, but he warns that you should be wary of making any personal statement too personal as this can end up pushing you into a niche within the profession.

It can also be helpful to ask colleagues about your qualities so you can highlight them in your CV. Including personal interests will give a sense that you have a life outside work and can maintain a work-life balance, so it is valuable to include them. This should be followed, finally, by your referees' details.

\section{What to avoid}

Try to avoid long paragraphs with large chunks of text. These can be off putting and will make your CV hard to read. Also make sure you check for spelling mistakes and other errors.

Including too much detail can deter the reader from reading on. Aim to cover around five years of clinical experience-information about GCSEs and A levels becomes irrelevant once you've achieved your medical degree.

Also, don't make claims that you can't live up to and that can't be demonstrated at interview. And make sure not to put anything in your $\mathrm{CV}$ that would compromise patient confidentiality.

\section{Making your CV stand out}

To make your CV more attractive to a potential employer, detail your actual achievements in your job, and how you've made a difference to patient care. This could be, say, measurable outcomes for patient care, or awards you've won. "Be specific, use examples, work out what your strengths are, and make sure you highlight them," advises Joanne Borbone, a consultant paediatrician based at Queen Alexandra Hospital, Portsmouth.

Ensure your CV is punchy, easy to read, and grabs the reader's attention. To do this, Martin Edwards, a consultant paediatrician based at the Children's Hospital for Wales in Cardiff, suggests you think of the details you include as "a bit like headlines in a news story that show what makes you different from everyone else."

You can also help your CV stand out by the way in which you present it. There are no hard and fast rules about this, but CVs should be easy to read, and using bullet points can help to emphasise the important points of your career. Redfern suggests that using a gentle highlight, such as grey or pale blue, for the section headers can improve the look and readability of your $\mathrm{CV}$ and make subject headings easy to identify.

It also helps to consider who will be reading your CV as part of a job application. You have to think about what they are looking for and adapt your CV to meet their requirements. And you need to make your CV fit the job you are applying for. Adrian James, registrar at the Royal College of Psychiatrists, says, "If you're applying for a post you can't just submit a generic $\mathrm{CV}$ - it has to be tailored to the job and what you're going to bring to the role."

\section{Keeping up to date}

Keep your CV up to date, and review it on at least an annual basis. Edwards suggests updating it "at the same time you are looking at your appraisal." He says for trainees the process is "fairly straightforward" and that they can update their CV at 
the end of the year at the same time as they tidy up their e-portfolio.

With so much information and experience to fit into a $\mathrm{CV}$, particularly by the time you've reached consultant level, there is always the danger of the document becoming overly long. More than 10 pages could be seen as excessive, but a CV that's too short lacks the detail to promote your skills and experience. It's important, therefore, to strike a balance, Edwards says.
To keep a CV concise and current, Redfern suggests showing it to a senior colleague who regularly interviews people, to help you to remove any unnecessary information as you go up the career ladder. Some of that information may be relevant to future applications, so he recommends keeping a CV archive which includes a history of your work experience. 